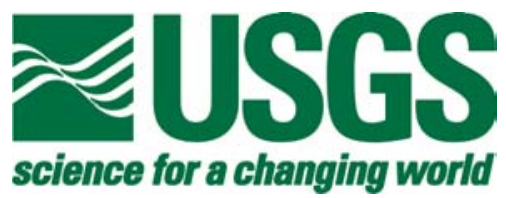

\title{
User's Guide for the MapImage Reprojection Software Package, Version 1.01
}

By Michael P. Finn and Jason R. Trent

Open-File Report 2004-1394

U.S. Department of the Interior U.S. Geological Survey 


\section{Contents}

Preface to Version 1.01 ...............................................................................1

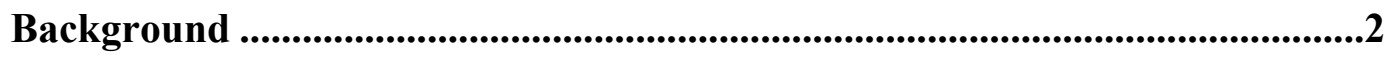

General Input Information ..........................................................................................3

Running mapimg ..................................................................................................4

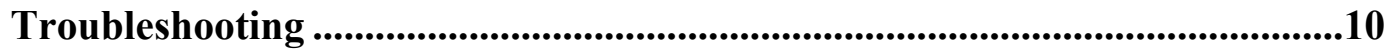

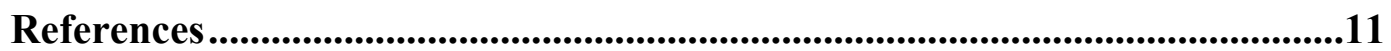




\section{User's Guide for the MapImage Reprojection Software Package, Version 1.01}

By Michael P. Finn and Jason R. Trent

\section{Preface to Version 1.01}

Major changes in MapImage version 1.01 include suggested bounds for parameters of each projection, support for larger input files, storage of last used input and output folders, and support for TIFF / GeoTIFF input images.

Included is a restatement of version 1.0 user's guide (Finn and Posch, 2003) with corrections for completeness and readability.

Any use of trade, product, or firm names is for descriptive purposes only and does not imply endorsement by the U. S. Government. 


\section{Background}

Scientists routinely accomplish small-scale geospatial modeling in the raster domain, using high-resolution datasets (such as 30-m data) for large parts of continents and lowresolution to high-resolution datasets for the entire globe. Recently, Usery and others (2003a) expanded on the previously limited empirical work with real geographic data by compiling and tabulating the accuracy of categorical areas in projected raster datasets of global extent. Geographers and applications programmers at the U.S. Geological Survey's (USGS) Mid-Continent Mapping Center (MCMC) undertook an effort to expand and evolve an internal USGS software package, MapImage, or mapimg, for raster map projection transformation (Usery and others, 2003a).

Daniel R. Steinwand of Science Applications International Corporation, Earth Resources Observation Systems Data Center in Sioux Falls, S. Dak., originally developed mapimg for the USGS, basing it on the USGS's General Cartographic Transformation Package (GCTP). It operated as a command line program on the Unix operating system. Through efforts at MCMC, and in coordination with Mr. Steinwand, this program has been transformed from an application based on a command line into a software package based on a graphic user interface for Windows, Linux, and Unix machines.

Usery and others (2003b) pointed out that many commercial software packages do not use exact projection equations and that even when exact projection equations are used, the software often results in error and sometimes does not complete the transformation for specific projections, at specific resampling resolutions, and for specific singularities. Direct implementation of point-to-point transformation with appropriate functions yields the variety of projections available in these software packages, but implementation with data other than points requires specific adaptation of the equations or prior preparation of the data to allow the transformation to succeed.

Additional constraints apply to global raster data. It appears that some packages use the USGS's GCTP or similar point transformations without adaptation to the specific characteristics of raster data (Usery and others, 2003b). It is most common for programs to compute transformations of raster data in an inverse fashion. Such mapping can result in an erroneous position and replicate data or create pixels not in the original space. As Usery and others (2003a) indicated, mapimg performs a corresponding forward transformation to ensure the same location results from both methods. The primary benefit of this function is to mask cells outside the domain.

MapImage 1.01 is now on the Web. You can download the User's Guide, source, and binaries from the following site: http://mcmcweb.er.usgs.gov/carto_research/projection/acc_proj_data.html 


\section{General Input Information}

Ensure that all INPUT files are in Generic Binary format or single-image TIFF or GeoTIFF format. No other file type is supported in the current version (1.01) of mapimg. If necessary, export your image into one of these formats before proceeding. It is highly recommended that all images are exported to Generic Binary format if necessary.

All TIFF/GeoTIFF images are exported to Generic Binary format as soon as they are loaded. TIFF formats store images as 32-bit color values, which are then extracted to Generic Binary. It is recommended that TIFF input only be used for thematic data; continuous data will lose all actual values, and only relative values will remain.

Band interleaved by line (BIL) is a storage format for image data. For a single band of data, BIL is the same as band sequential (BSQ) and band interleaved by pixel (BIP) (Slater, 1980). Generic Binary, as we use it (consistent with ERDAS Imagine), is any of these single-band formats that store data in a left-to-right, top-to-bottom row order (ERDAS, 1999). It does allow for varying data types-for example, from 8 to 64 bit.

CAUTION: The Generic Binary file must use the same byte ordering (endian) as the computer running mapimg. The file is a raw image with absolutely no metadata, not even the dimensions of the image, which must be known by the user. The output also will be in the same raw format.

A common naming convention is recommended to keep track of your files: in particular, the format (type)_(proj)_(pix_size).img, where type represents the type of file being used, proj represents the projection used, and pix_size represents the output file's pixel size. For example, you would use glc_sine_30sec.img to represent a global land cover image in the sinusoidal projection with a pixel size of 30 arc-seconds. Notice that the file name always ends in .img, signifying a generic binary image. Although these conventions are not mandatory for proper program execution, they provide a useful and reliable way to keep track of your files.

Anytime mapimg is run on a file, an associated info file will be created from the parameters selected by the user for both the input and the output image. For an input/output file called filename.img, the associated .info file will be named filename.img.info. It is not absolutely necessary to save these files, but it is highly recommended. If the input/output files are to be used again in the future, the .info files will make sure the correct projection parameters are used. These ".info" files function as a type of metadata.

This version of mapimg, 1.01, does not include every major Global (small-scale) projection. In addition, Universal Transverse Mercator and State Plane projections, because they can be considered local or regional (large-scale), currently are not supported. These projections may be supported in a later version. GeoTIFF formatted output also may be supported in a later version. 


\section{Running mapimg}

MapImage is a stand-alone program available for Windows, Linux, and Solaris. All images are of the Windows version, but Linux and Solaris versions will look similar. Double click the mapimg executable (mapimg.exe) to begin execution of the program. Upon startup, the dialog box shown in figure 1 will appear.

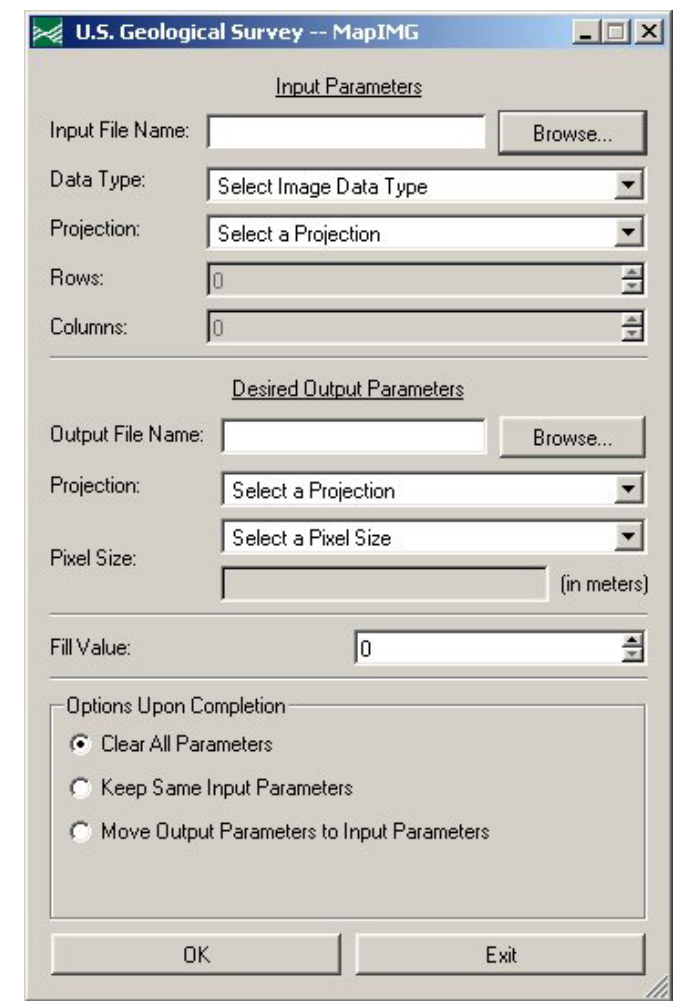

Figure 1. Startup screen.

To perform your projection transformation, complete the following steps:

\section{(1) Setup the Input Parameters:}

a. Select an appropriate Input File Name by using the Browse button and navigating to the appropriate file or by typing the appropriate path and file name. This file MUST be in Generic Binary or a TIFF format. If required parameters can be determined, the input parameters will be set for you, and you may skip to step $2 \mathrm{a}$.

b. Select the Data Type of the input file from the Data Type Drop Down Menu (fig. 2). 


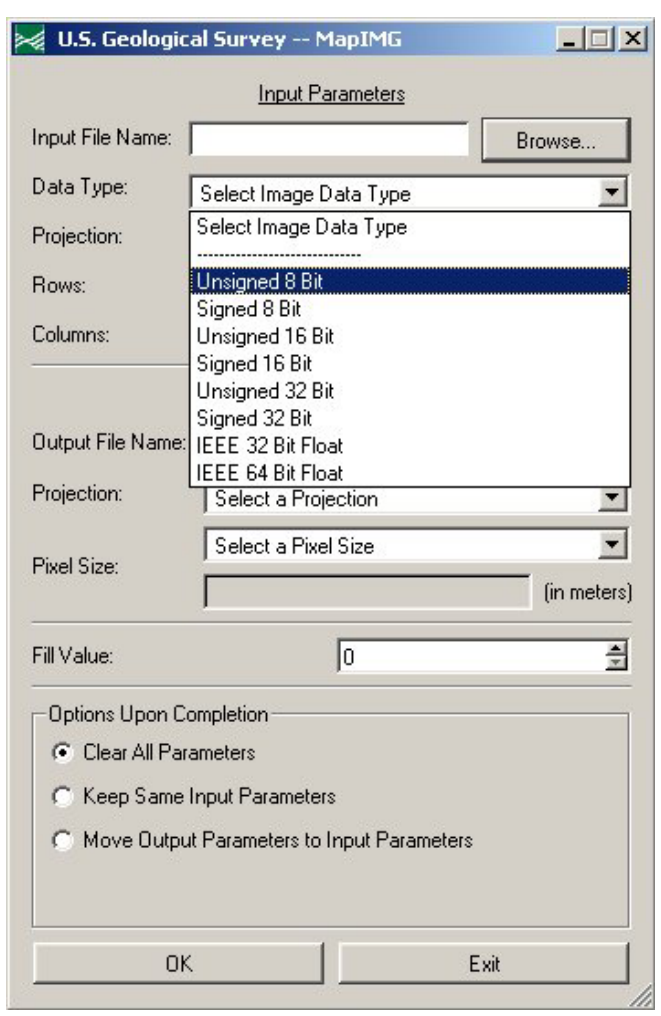

Figure 2. Data Type drop down menu.

c. Select the Projection that your input file represents from the Projection Drop Down Menu (fig. 3).

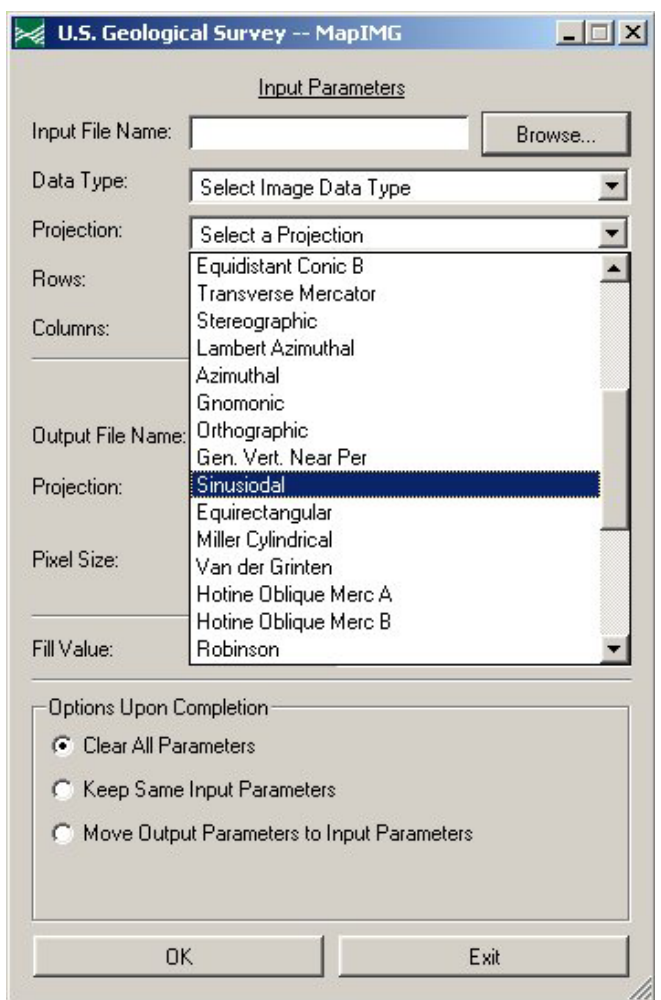

Figure 3. Projection drop down menu. 
After a projection has been selected, a secondary dialog box such as the following may appear (fig. 4). If this screen does not appear, an associated .info file has already been found for this input file.

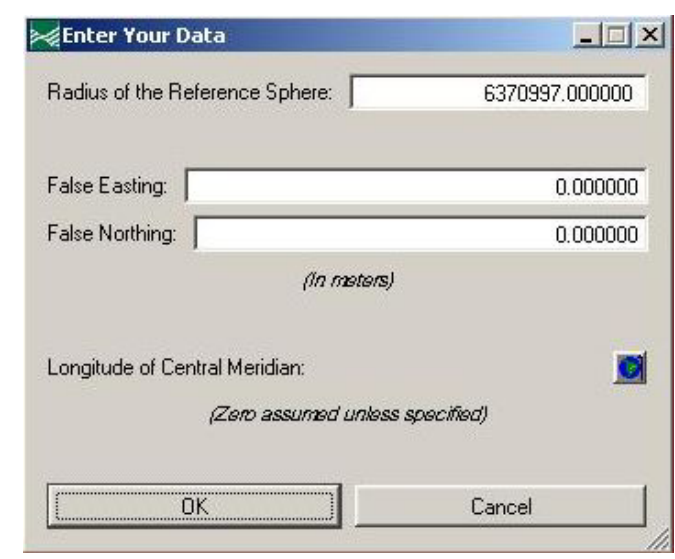

Figure 4. Secondary dialog.

An example of a Sinusoidal projection is shown in figure 4. It is in this dialog that you may make all changes to reflect the proper parameters of your input file. In this instance, the Radius of the Reference Sphere, False Easting, and False Northing may be changed by entering a new value into the respective edit boxes.

To change the Longitude of Central Meridian, click the globe button. This will bring up the dialog shown in figure 5.

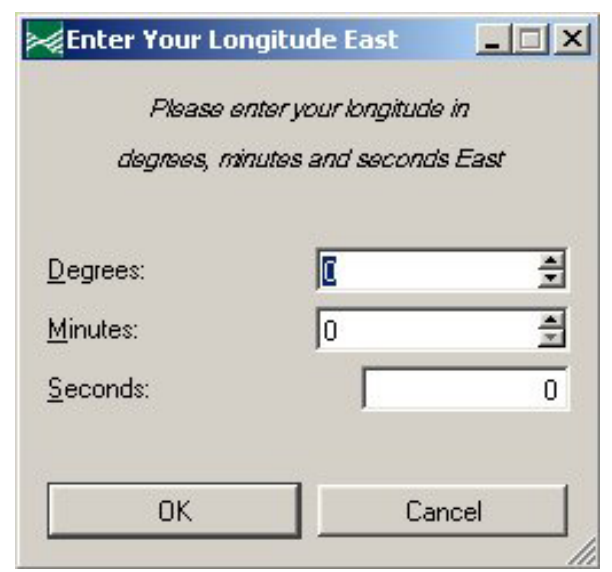

Figure 5. Longitude input screen.

At this time, the Degrees, Minutes, and Seconds of the desired longitude may be entered. Press $\boldsymbol{O K}$ to accept the values or Cancel to ignore the change. A similar process can be used for entering latitudes as well.

Once the changes have been either accepted or ignored, the previous dialog (fig. 4) will again become active. At this time, press $\boldsymbol{O K}$ to accept the changes, or Cancel to ignore the changes. 
d. If necessary, input the number of rows/columns; the Number of Rows and Number of Columns boxes will be activated (fig. 1). To input the Number of Rows/Columns, simply enter the values into the appropriate boxes. This information is available in the ".info" file or from the original metadata.

\section{(2) Set up the Desired Output Parameters:}

a. Create an appropriate Output File Name by using the Browse button to navigate to an appropriate save location. To create a file with a new name, simply enter the name and press Save. To overwrite an existing file, simply click the file to be overwritten and press Save. A file name also may be typed manually into the edit box. The output image will be in the same raw data format as the input. Also, the output will have the same data type. However, the dimensions may change depending on which projection and pixel sizes are used.

b. Select the Projection of the desired output projection from the Projection Drop Down Menu. Secondary dialogs similar to those previously seen will be available. In this instance, the secondary dialogs can be used to make any changes that need to be reflected in the output file.

c. When the secondary dialogs have been completed, the main dialog will become active again. Select an output file Pixel Size from the Pixel Size Drop Down Menu (fig. 6).

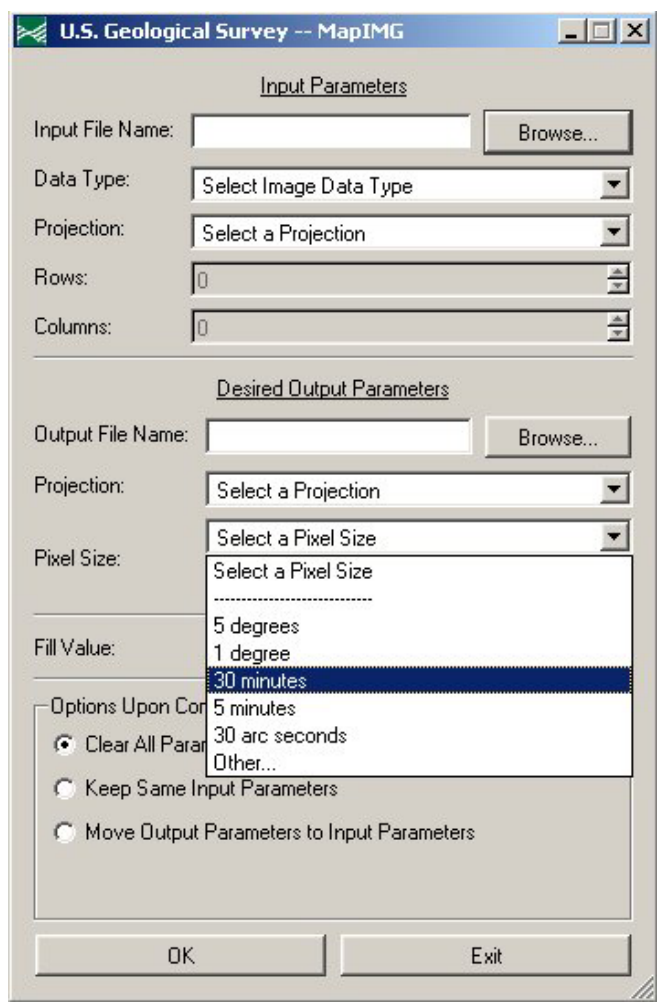

Figure 6. Pixel Size drop down menu. 
Predetermined options are available for 5 degrees, 1 degree, 30 minutes, 5 minutes, and 30 arc-seconds. If Other is selected, the Pixel Size Text Box (fig. 1) will be activated and any other size may then be entered. It is important to note that this value may be entered only in meters.

(3) Assign a correct Fill Value. The Fill Value may be any value that currently is not being used as a data value. Experience has shown that for categorical data, the fill value can be calculated as follows:

(Number of input file classes $+n$ ), where $n=$ any integer $>0$ The recommended value for $\mathrm{n}$ is 2 .

For example:

\begin{tabular}{llr}
\multicolumn{2}{c}{ Category Number } & \multicolumn{1}{c}{ Category Name } \\
0 & Background & Histogram \\
10 & Urban & 39042 \\
41 & Mature Deciduous & 363609 \\
42 & Mature Planted Pine & 17172 \\
43 & Mixed Deciduous / Pine & 20655 \\
50 & Water & 14823
\end{tabular}

In this case, the highest category number is 50 . The recommended fill value would be 52.

(4) Choose your Options Upon Completion (fig. 1).

a. Choose Clear All Parameters to clear all information once the reprojection is completed. This is the default selection.

b. Choose Keep Same Input Parameters to leave the current input parameters active. This option is useful when performing multiple reprojections on the same input file.

c. Choose Move Output Parameters to Input Parameters to make your created output parameters your new input parameters. This option is useful when performing a multiple reprojection. For example: Geographic $->$ Sinusoidal $->$ Mercator $->$ Hammer.

(5) Press $\boldsymbol{O K}$ to perform the transformation. Once started, the transformation can be aborted by pressing the Abort button (fig. 7).

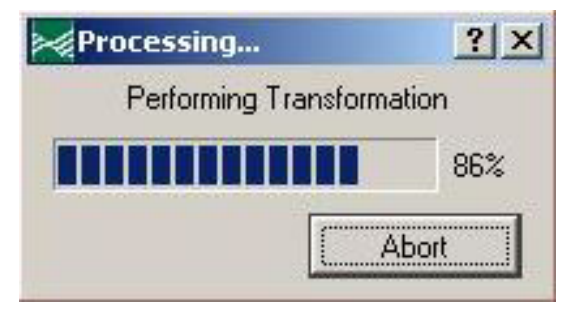

Figure 7. Abort screen. 
If the transformation is allowed to finish, the Output Rows/Columns will be shown. It is important to record these values for later use (fig. 8).

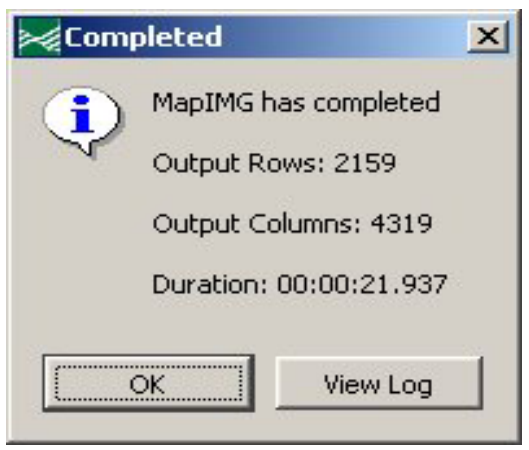

Figure 8. Output Rows/Columns.

(6) You may now choose to View Log (fig. 8). This will show all messages generated by the reprojection including parameters and any errors that may have occurred (fig. 9). The $\log$ may be saved by clicking the Save... button.

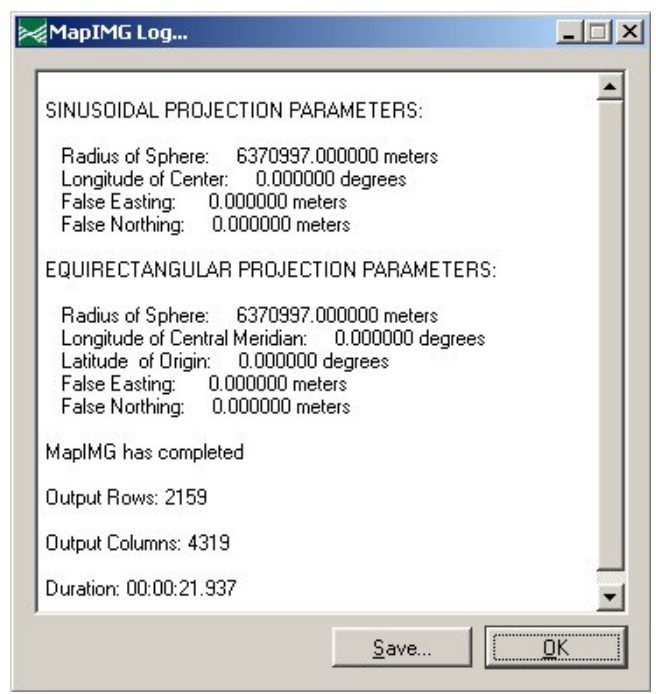

Figure 9. Log Output

NOTE: Not all error messages indicate an error in output. Out of Area and other similar messages indicate where pixels are out of viewing area and should not be included in the new projection.

(7) Repeat process for other files if desired. 


\section{Troubleshooting}

Listed below are some common errors that may occur and possible remedies.

MapIMG - Overwrite file?

- Output image or .info file already exists for the name specified. Choose "Yes" to overwrite and continue or "No" to cancel.

This projection is not currently supported!

- The projection you've selected currently is not available.

- Please check the Web site periodically for newer versions of mapimg with additional projections.

An internal error has occurred while trying to open designated output file.

- Check that the output file and associated .info file do not exist and are not write protected.

- Make sure that there is enough available disk space for reprojection.

An internal error has occurred while trying to open (read) the designated project file.

- Check that the input file exists.

- Make sure that there is enough available disk space for reprojection.

An internal error has occurred while trying to allocate the input image buffer!

- There currently is not enough free memory to execute the projection. Try freeing memory by using a combination of the following:

1. closing unnecessary programs

2. selecting a larger output pixel size

3. using a smaller input image 


\section{$\underline{\text { References }}$}

ERDAS Inc., 1999, ERDAS Field Guide. Fifth Edition: Atlanta, Ga.

Finn, M.P., and Posch, S.T., 2003, Users Guide for the MapImage Reprojection Software Package: U.S. Geological Survey Open-File Report 03-433.

Slater, P.N., 1980, Remote sensing: Optics and optical systems. Reading, Mass., Addison-Wesley Publishing Company, Inc.

Usery, E.L., Finn, M.P., Cox, J.D., Beard, T., Ruhl, S. and Bearden, M., 2003a, Projecting global datasets to achieve equal areas. Cartography and Geographic Information Science, v. 30, no. 1., Washington, D.C., ACSM, p. 69-79.

Usery, E.L., Seong, J.C., Steinwand, D., and Finn, M.P., 2003b, Accurate projection of small-scale raster datasets. Proceedings $21^{\text {st }}$ International Cartographic Conference, Durban, South Africa. International Cartographic Association. 